Eucalyptus oil, as used within its usual dose limits of $\frac{1}{2}$ to 3 minims, seems never to give rise to any toxic symptoms, but a remedy such as this, which has almost become a household stand-by for catarrh and the like, bas, in not a few cases, been taken by mistake in larger doses, and in some cases with poisonous effect. The number of such cases is as yet hut small (in fact scarcely over a dozen) and their record is naturally fragmentary, as all notes of poisoning are apt to be when the necessity of therapeutic measures must perforce prevent detailed stady of the resulting clinical phenomena. In these cases of eucalyptus poisoning the symptoms have varied greatly according to the amount of oil taken, and apparently also according to the idiosyncrasies of the patient. Incidentally it may be mentioned that Davies ${ }^{18} \mathrm{knew}$ a patient who fainted even with the smell of it.

In general, toxic symptoms have been noted after doses of 1 drachm or more, but $H$. S. Taylor ${ }^{11}$ speaks of a patient who felt a little giddiness and confusion after $\frac{1}{2}$ drachm. Most of the cases, however, developed marked toxic symptoms after doses of $1 \mathrm{drachm}$ or more, as in those of Taylor, ${ }^{11}$ Benham, ${ }^{12}$ the two of Atkinson, ${ }^{18}$ a case of Allan's, ${ }^{20}$ one case of Kirkness, ${ }^{19}$ and the above case. In other cases the dose of eucalyptus oil taken was 2 drachms, as in that of $0 r{ }^{16} 3$ drachms or even 6 drachms, as in the cases of Benjamin 14 and Myott. ${ }^{15}$

With such different dosage the time of onset of toxic symptoms has naturally varied greatly-from ten minutes in one case to one or two hours in another. In like manner the kind of symptoms and the order of their development have shown differences. Vomiting was gene. rally the first sign-in the case reported by Kunkel ${ }^{9}$ it was the only one-and it was frequently followed by abdominal pain and diarrhoea. In most of the cases, whether preceded by gastro-intestinal irritation or not, unconsciousness, varying in degree from mere drowsiness to coma, supervened.

In some the first symptom noticed was a giddiness, as in the cases of Kirkness, ${ }^{19}$ Sheaf, ${ }^{8}$ and Taylor. ${ }^{11}$ In all but the slightest cases further effects manifested them. selves in pallor, quick pulse, and general collapse, as well as frequently respiratory disturbance. No after-effects were noted beyond the temporary odour of the breath.

In most recovery with or without medical aid was quick. Generally the early vomiting removed a-large amount of the poison, and in the cases where only cerebral symptoms were present the use of an emetio led to a speody recovery. Hence an emetic, if required, and afterwards stimulant treatment, seemed in almost all the cases to give the necessary relief. The duration of the toxic symptoms in the cases recovering varied from an hour or two on an average up to ten hours in a case recorded by Benjamin, ${ }^{14}$ where a boy ill with remittent fever had got by mistake 6 drachms.

Nevinny ${ }^{6}$ in 1898 mentioned that a fatal result had not yet been observed, bat since then several have been put on record. In one reported by Lewin, ${ }^{b}$ a boy of 10 , after taking 15 grams, suffered from vomiting, pallor of the lips, rapid weak pulse, sighing respiration, and air hunger, and died in fifteen minuteg. At the post-mortem examination, blood was found in the plearal cervity. In a case of Myott's, ${ }^{15}$ a man of 34, after taking 4 drachms becsmo soon dazed and later unconscious. The pulse becsme quick and the breathing laboured. The physical signs pointed to congestion throughout both lungs. He rallied a little, bat died within forty hours after taking the oil. The post-mortem examination showed inter alia reddening of the trachea and bronchi. Nealo also reports a fatal case.

The explanation of these toxic cases has not so far been satisfactory. Schalz ${ }^{1}$ says that pure encalyptus oil is very slightly active, and that 10 grams produces no effect, while Cathbert Hall ${ }^{10}$ considers that encalyptol-the chief constituent-has probably very little toxic result. Manquat ${ }^{8}$ quotes the experiences of Bouveret and Peschade with the subcutaneous injection of encalyptol in liquid vaseline, where the patients sometimes felt a heat in the abdomen, and at other times showed symptoms of cerebral phenomena of a more or less uncomfortable nature.

Siegen, as Manquat mentions, noted with a dose of $3 \frac{1}{2}$ grams headache and mental prostration. The other phenomena are like those produced by turpentine-coma, weakness of reflexes and of the respiration, lowering of the blood pressure and temperatare, and in fatal dose respiratory paralysis. Kunkel, ${ }^{9}$ in discussing his case, considers it may have been caused by another kind of encalyptus oil, or else that it might be due to an adulteration.

REFERENCES.

1 H. Bchulz, Das Eucalyptusöl, Bonn, 1881. ${ }^{2}$ F. J. Owen, Austral. Med. Journ., Melbourne, 1885, N.S. vii, 394-7. ${ }^{3}$ E. Sheaf, BRimist MEDICAI JoURNAL, 1888, i, 849. A. Neale, Australas. Med. Gaz. Bydney, 1893, xil, 115. L. Lewin, Lehrbuch der Toxikologie, 2te Auf.

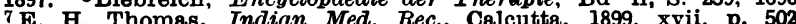
8. H. Thomas, Indian Med. Rec., Calcutta, 1899, Xvil, $^{\mathrm{D} .} 502$ 9 A. J. Kunkel, Handbuch der Toxikologie, 1901. $10 \mathrm{C}$. Héll, On Aucalyptunkel, Handbuch der Toxikologie, 11 191. 10. Hail, On Eucalyptus Oils, M.D. Thesis, Sydney, 1904. 11 H. 8. Taylor, Lancet, London, 1905 , ii, p. 963. 12 F. L. Benham, Lancet. London, 1905, ii p. 1894. 13 H. R. Davies, Lancet, London, 1906, i, 403. 14 J. Benjamin Indian Lancet, Calcutta, 1906, Xxvii, 720, also Lancet, is London, 1906 BRITISH MEDICAL JoURNAL, i. 1906, p. 558 . 16 J. Orr, ibid., 1906, p. 1085 . 17 Bchroeder. Atrztl. 8achverst. Z tg., Berlin, 1908, xiv, 153. 18 T. R. Atkinson, BRITISH MEDICAI JoURNAI, December 12th, $1909.19 \mathrm{~W}$. R. Kirkness, ibid., January 29th, 1910. ${ }^{20}$ J. Allan, ibid., March 5th, 1910.

\section{THE CURATIVE EFFECT OF SALVARSAN} (“606") IN CASES OF FRAMBOASIA.

By HENRY ALSTON, M.B., GOVERNMENT MEDICAL OFFICER, TRINIDAD.

THE following experiments were carried out at the St. Augustine Yaws. Hospital, Trinidad, from January 4th onwards. On that day Dr. Rost, the surgeon of the German cruiser Freya, who had some tubes of "606" which had been given to him at Kiel, at my reguest kindly injected five cases of yaws, three adults and two children. Dr. Rost used 0.6 gram ( 9 grains) in an emulsion with 5 to $6 \mathrm{c.cm}$. of sterilized olive oil for adults. The injection was made deeply into the muscle of the buttock. In twenty-four hours a marked improvement was noticeable, the yaws tubercles drying up from the periphery, so that whitish circles appeared to be surrounding them.

The improvement continued, and on January 8th I decided to try an experiment. I had read that in the case of an injected syphilitic mother and suckling the baby was cured by suckling the mother; and I was also aware of Professor Ehrlich's view that "606" created an antibody by rapidly destroying spirochaetes. I therefore put a cantharides blister on two of the cases being cured by the " 606 " treatment, and injected the serum from the blister into several cases of yaws, the adult dose being $16 \mathrm{c.cm}$. To my astonishment, the serum acted as rapialy as "606." In some cases improvement was noticed in sixteen hours after injection. On January 11th, at my request, Dr. Rost injected thirteen more cases of yaws with "606," and the same rapid improvement was noticeablo - in some cases as early as sixteen hours after. I applied fly blisters to some of these cases and used the serum for more experiments. In every case injected with serum I got a rapid improvement.

I ought to inject the serum cases every seven or ten days; but I am leaving them alone to see how long the improvement after a single dose of serum will last.

On January 17th the Yaws Hospital was visited by the Acting Surgeon-General, Dr. J. W. Eakkin, Dr. Rost, and two of my confrères, who saw all the cases under treatment both with " 606 " and seram, and who also saw the detailed account in my book.

I believe that this is the first instance known of a drug producing an antitoxic serum with curative properties, and the effect, in a boy, of injecting 2 drachms and 20 minims of a serum that had not been hyper-immunized, was very astonishing.

But a greater astonishment was to come. I pat a cantharides blister on two cases that were being cured by the serum from a " 606 " case, and in both an improvement was noticeable in twenty-four hours. Being boys, each received $8 \mathrm{cccm}$. of serum. Whether the effect is due to the drug or to an antitoxin, the dilution is very great in what I call the secondary serum cases.

It occurred to me that the results I obtained with serum would be explained if the failure of a cure by Nature was due to the absence of complement in the blood of yaws patients. I argued that it was possible that the antibody 
required to be united with complement in order to be an antidote, and that the complement was "fixed" by the toxin. If so, all that was required was to supply complement. I performed three experiments :

1. I pat a fly blister on some healthy persons, and injected normal human serum into yaws cases. No result tollowed.

2. I heated the serum from cases that were being cured after " 606 " injections, and the serum, even after boiling, was efficacious.

3. I put blisters on three cases of yaws, and used the patients' own serum for their injection. No result.

I have therefore abandoned the provisional hypothesis of fixation of the complement by the toxin.

I also performed the following control experiments: I injected sterilized water with tincture of cantharides, and satisfied myself that the presence of cantharides in the serum has no effect whatever.

I injected four cases with cacodylate of soda for com. parison, and I see nothing to compare with the experiments with " 606 " and the serum.

A farther experiment with normal human serum obtained by a blister was negative.

It is my intention to inject a goat with "606," and use the goat's milk for young children with yaws. I will also blister cases that have been cured of yaws under ordinary treatment and experiment with their serum. If it be possible to cultivate spirochrotes, I suggest that spirochates be injected into a horse; that this be followed by " 606 " injections, and that experiments be made with the serum in cases of syphilis.

When the experiments were carried on, no other treatment of any kind was used; even a bath was not per mitted. No complications, locel or general, occurred. I believe that the dose of " 606 " for yaws can be safely increased, as the reaction was very slight, and in some cases absent. Cases will require a second injection. The pain after the injection of the emulsion lasts for about six hours, but is not very great. Dr. Rost intends to publish the cases injected by him with "606." We all owe him a debt of gratitude for so willingly using his " 606 " tubes in yaws cases. The blisters were applied on the fourth day atter injection with "606," or with the serum of a "606" case.

In the light of marked effects in the secondary serum cases we may have to change the hypothesis of the formation of an antibody by the injections. The curative substance seems to have the power of increasing itself. Is it a ferment?

\section{A REPORT ON THREE OASES TREATED WITH EHRLICH'S SPECIFIC FOR SYPHILIS.}

\section{BY}

E. G. FFRENCH, M.D., F.R.C.S.Ed., CAPtaIn R.A.M.C., STATION HOSPITAL, AHMEDNAGAR, SOUTHERN INDIA.

Throdgr the kindness of Mr. J. E. R. MoDonagh of the London Look Hospital, who spared me a few tubes of "606," I have been able to carry out Ehrlich's treatment in three selected cases of syphilis. I had the good fortune to see Mr. MoDonegh treat some petients in London, and I have endesvoured to treat the cases after his method. The doses were received in the form of a yellow powder hermetically sealed in glass anpullae, the dose ranging from 0.3 to 0.9 gram. One end of the glass ampulla is broken off, and the contents tarned out into a glass mortar which has been sterilized. The powder is dissolved first of all in as small a quantity of ethyl alcohol as possible. To this is added about $10 \mathrm{c.cm}$. of very hot water, and the fluids stirred vigorously until every trace has been dissolved; then a small quantity of solution of sodium hydrate (40 grams to the litre) is added, well mixed, and three drops of a $\frac{1}{2}$ per cent. alcoholic solation of phenolphthalein added, and titrated with normal acetic acid until the red colour has completely disappeared. Finally, a few drops of normal sodium hydrate are added until a faint rose tint comes back to remain, so that the solution is just alkaline. The solution is now ready for injection. A fairly large syringe capable of holding $12 \mathrm{c.cm}$. and fitted with a long needle is used. It is necessary to have a needle with a large bore, as sometimes the fluid is apt to become viscid, and would certainly block an ordinary needle.

The urine must be tested in every case before injection and daily afterwards. The strictest antiseptic precantions should be taken and the needle boiled in salad oil to prevent the blunting which occurs with water.

The scapular region is a suitable site for injection. The skin over the scapular muscles is well washed with soap and water, nothing else being used. The contents of the mortar are drawn into the syringe, and the needle planged deeply into the muscles of the scapula on the side selected. The fluid is then slowly but firmly driven into the muscles. The needle is then slowly withdrawn, and a piece of aseptic gauze held by rubber plaster put over the punc. tare. The patient should be kept in bed for five or six days.

\section{CASE I.}

Colour-Sergeant G., aged 35 years, acquired syphilis eleven zears ago. A very short time afterwards he had seventy-two days' treatment with hyd. $\bar{c}$ cret. in hospital. He did not have any further treatment, and kept fit until his tonsils and palate became ulcerated six weeks ago.

On admission to huspital he was found to be suffering from a large perforation of the soft palate and extensive ulceration of the tonsils and posterior pharynx. The avula had already ulcerated away.

Five days afterwards 0.5 gram of " 606 " was injected into the muscles of the right scapular region. He did not have any rise of temperature, and felt no ill effects, with the exception of some slight soreness at the sight of injection.

Two days afterwards uthere was a distinct improvement in the appearance of the ulcers. The sloughs had disappeared, and the perforation looked smaller. At the end of the first week the tonsils and posterior pharynx had healed completely and the perforation was about one-third the original size. He was weighed, and found to have gained $5 \mathrm{lb}$. About this time a small amount of lymphoid tissue was noticed at the site of the uvula.

A fortnight afterwards the hole in the palate had almost closed, and the patient's general condition was greatly improved.

Four weeks afterwards the perforation had closed completely, and the lymphoid tissue at the site of the uvula had grown into a fairly large process. He had regained his normal voice, and on being weighed was found to have gained $16 \mathrm{lb}$.

\section{Case II.}

Lance-Corporal R., aged 32 years, acquired syphilis five years ago. He did not receive any mercurial treatment internally, as the sore was diagnosed to be soft. He kept fit until May, 1910, when he was admitted to hospital suffering from severe ulceration of the upper gums, tonsils, and posterior pharynx. He had also diserse of the nasal bone on the left side and wellmarked tibial pains at night. He was given several courses of inunction periodically, but his condition did not improve.

He was sent to me as a selected case for " 606 " treatment. I found that he was suffering from the above-mentioned symptoms, and that quite recently he had lost five upper teeth on the right side. A few days afterwards 0.5 gram was injected into the muscles of the right scapular region. He had no pain afterwards, but had a rise of temperature for three days, the highest being $101^{\circ}$.

Two days afterwards the tibial pains ceased and the condition of the gums and throat was markedly improved. At the end of the first week the throat had completely healed and the gums were almost well, the teeth being quite firm. His weight had increased $2 \frac{1}{3} \mathrm{lb}$.

A fortnight afterwards the gums and. throat were quite healed; a piece of necrosed bone came away from the left nostril, and the discharge, which was profuse before, was greatly diminished.

On the eighteenth day he had gained over 1 st. in weight and looked quite fit. He is now quite free from any lesions.

\section{CASE III}

Private L., aged 28 years, acquired syphilis in October, 1910 He was admitted to hospital at Poona soon afterwards and developed a profuse papular rash over the face, neck, trunk and upper and lower. limbs. He was given three courses of ung. hydrarg., and was transferred here for "606" treatment, as his condition was not improving. On admission, he had the rash over the face, trunk, and limbs, some ulceration of both tonsils, and well-marked enlargement of the cervical and inguinal glands.

He was injected in the right gcapular muscles with $0.5 \mathrm{gram}$ 606." He suffered slight pain in the muscles for about two hours afterwards. Two days afterwards the enlarged glands had completely disappeared, and the rash was fading rapidly from the face. The condition of the throat showed marked improvement. He had a rise of temperature for three days. At the end of a week the rash had completely disappeared from the face, the throst was quite well, and the rash was fading from the trunk and limbs. He had gained 1 th $\mathrm{lb}$. in weight. Eighteen days afterwards, with the exception of a few stains on the gkin the result of the rash, there was nothing to show that he had ever had syphilis. He had gained $8 \frac{1}{2} \mathrm{lb}$. in weight.

The cases described are the first to be treated in India with Ehrlich's wonderful specific. 